\title{
Autonomous Battery Exchange of UAVs with a Mobile Ground Base
}

\author{
Éamon Barrett, Mark Reiling, Seyedmohsen Mirhassani, René Meijering, Jeroen Jager, Nicola Mimmo, \\ Flavio Callegati, Lorenzo Marconi, Raffaella Carloni, and Stefano Stramigioli
}

\begin{abstract}
This paper presents the autonomous battery exchange operation for small scale UAVs, using a mobile ground base that carries a robotic arm and a service station containing the battery exchange mechanism. The goal of this work is to demonstrate the means to increase the autonomy and persistence of robotic systems without requiring human intervention. The design and control of the system and its components are presented in detail, as well as the collaborative software framework used to plan and execute complex missions. Finally, the results of autonomous outdoor experiments are presented, in which the ground rover successfully localizes, retrieves, services, and deploys the landed UAV, proving its capacity to extend and enhance autonomous operations.
\end{abstract}

\section{INTRODUCTION}

Autonomous robotic platforms are increasingly utilized to execute monitoring [1], inspection [2], search and rescue [3], or disaster response missions [4]. Just as their assignments are growing more numerous and complex, the autonomous capabilities of the robotic system handling these tasks need to evolve with them, to enable new applications and to relieve their human operators of menial or dangerous work.

The work presented in this paper was conducted in the context of the European project SHERPA [3], in which a mixed ground and aerial robotic platform with high degree of autonomy supports human rescuers in search and rescue operations in hostile environments. This paper focuses on the autonomous battery exchange of small-scale quadrotor UAVs, which enables them to carry out persistent missions without relying on human operators to recover or recharge

This work was funded by the European Commission's Seventh Framework Programme as part of the project SHERPA under grant no. 600958.

E. Barrett was with the Department of Electrical Engineering, Mathematics and Computer Science, University of Twente Enschede, The Netherlands. He is now with the Department of Advanced Robotics, (Fondazione) Istituto Italiano di Tecnologia, Genova, Italy. (e-mail: eamon.barrett@iit.it)

M. Reiling and R. Meijering were with the Department of Electrical Engineering, Mathematics and Computer Science, University of Twente Enschede, The Netherlands. They are now with the Saxion University of Applied Sciences. (e-mail: \{m.reiling, r.meijering $@$ @ saxion.nl)

J. Jager was with the Department of Electrical Engineering, Mathematics and Computer Science, University of Twente, The Netherlands.

S. Mirhassani, N. Mimmo, F. Callegati, and L. Marconi are with the Department of Electrical, Electronic and Information Engineering "Guglielmo Marconi" (DEI), University of Bologna, Italy. (email: \{seyedmohs.mirhassani@unibo.it; nicola.mimmo2, flavio.callegati2, lorenzo.marconi@unibo.it)

R. Carloni was with the Department of Electrical Engineering, Mathematics and Computer Science, University of Twente, The Netherlands. She is now with the Faculty of Science and Engineering, University of Groningen, The Netherlands. (e-mail: r.carloni@rug.nl)

S. Stramigioli is with the Department of Electrical Engineering, Mathematics and Computer Science, University of Twente, The Netherlands and also with the Biomechatronics and Energy-Efficient Robotics Lab, ITMO University, Saint Petersburg, Russia. (e-mail: s.stramigioli@utwente.nl)

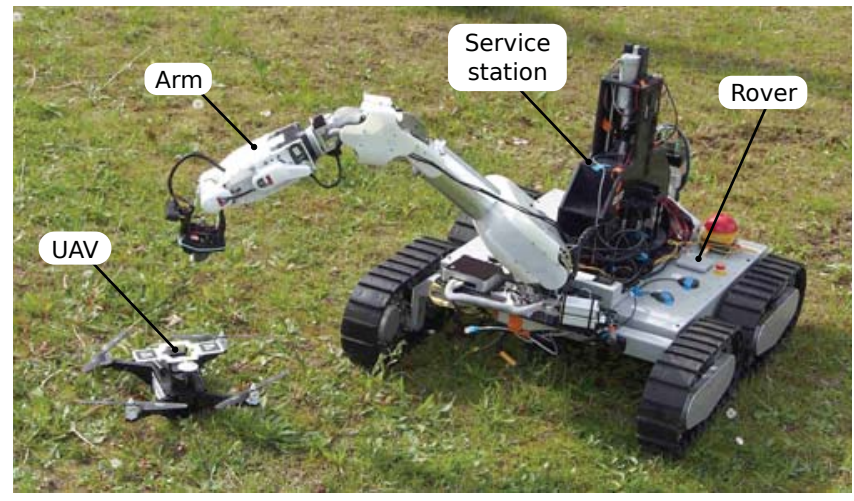

Fig. 1: The SHERPA mobile ground base for autonomous battery exchange

them. In this scope, we propose a mobile service station, mounted on a ground rover and equipped with a robotic arm to retrieve the UAVs, as shown in Figure 1.

In the following we outline the state of the art and motivate the main design choices for providing the supportive infrastructure for the small scale UAVs.

1) Power Replenishment: The UAV's energy supply can be replenished either by recharging or by exchanging the depleted batteries. Several design concepts for both recharging and exchanging the batteries are in [5].

Automated battery recharging stations are the most common solution due to their lower complexity, but also show a lower vehicle utilization due to the long recharging time [6] [8]. On the other hand, a battery exchange station needs a mechanism that can extract and replace the batteries from the UAV, as well as a storage mechanism to hold the spare batteries, but requires no additional time to charge the batteries before the UAV is operational again, which typically takes several times the flight time. The UAV needs to be designed such that the battery is easily exchangeable but still mounted well enough to withstand the vibrations experienced in flight and landing. Battery exchange stations, that combine rotating battery magazines with at least one linear actuator to move the battery containers from the UAV into the magazine and vice versa are presented in [9]-[11], while [12] uses linearly arranged battery bays.

As analyzed in [5], recharging stations are more economical for low coverage scenarios in terms of the provided coverage, i.e., how many UAVs are operational at a given time, versus the total system cost. Nonetheless, we chose a battery exchange system to minimize the amount of UAVs and service stations to be transported to the mission areas.

2) Service Provision: To service the UAVs in a dynamic 
mission with shifting areas of interest and, thus, to extend their operational radius, we chose a mobile ground base, as the strategical placement of stationary service stations across the operational environment [6], [13] is not a practical solution for search and rescue or emergency response operations. In [7], [8] recharging stations have been mounted on commercial mobile ground vehicles, but these platforms were intended for laboratory environments. Instead, a tracked vehicle with robust outdoor navigation capabilities was developed for the SHERPA project (BlueBotics SA, Switzerland).

3) UAV Retrieval: The service stations presented in the literature require the UAV to precisely and safely land on a landing pad on the station. Most of the time, however, the landing precision of the UAV is not sufficient to engage the replenishment mechanism, also due to wind. This is especially the case for battery exchange mechanisms, where a close mechanical fit is required. To overcome the alignment issue some of the proposed stations have passive guidance systems that function like funnels [5], [10], while others are equipped with active alignment systems, like small arms or wire mechanisms [11], [13] that position and secure the UAVs during replenishment. However, many systems also still rely on external position sensing in order to land the UAV on the platform.

While these systems alleviate the problem of retrieving the UAVs, the landing operation is still delicate. The SHERPA mission requires that the robotic platform can reliably retrieve the UAVs without human involvement. Hence, we chose to mount a robotic arm on the ground rover, that is capable of robustly retrieving, docking, and deploying the UAVs. In order to increase robustness during the manipulation and docking of the UAVs, the arm is designed with a variable mechanical compliance.

4) Software Framework: Complex missions involving a heterogeneous robotic team also necessitate a suited control and communications structure [14], [15]. A framework for the automatic specification, generation, and execution of high-level collaborative mission plans has been presented in [16] as part of the SHERPA project, which is a more robust and flexible solution than systems based on a central planning and scheduling algorithm [6], [17].

The remainder of this paper is organized as follows. Section II outlines the software framework used by the agents of the system to plan and execute tasks, while Section III presents the design of these agents in detail. Experimental results on the autonomous battery exchange are presented in Section IV, and concluding remarks are drawn in Section V.

\section{Collaborative Mission Framework}

Complex collaborative tasks that would be difficult or impossible to perform for individual agents require the cooperation and coordination of the participating agents. This section presents the distributed communication and control architecture, that enables a larger heterogeneous humanrobot team to effectively work together in a robust and versatile manner, even under adverse operating conditions. The delegation framework and semantic structures developed

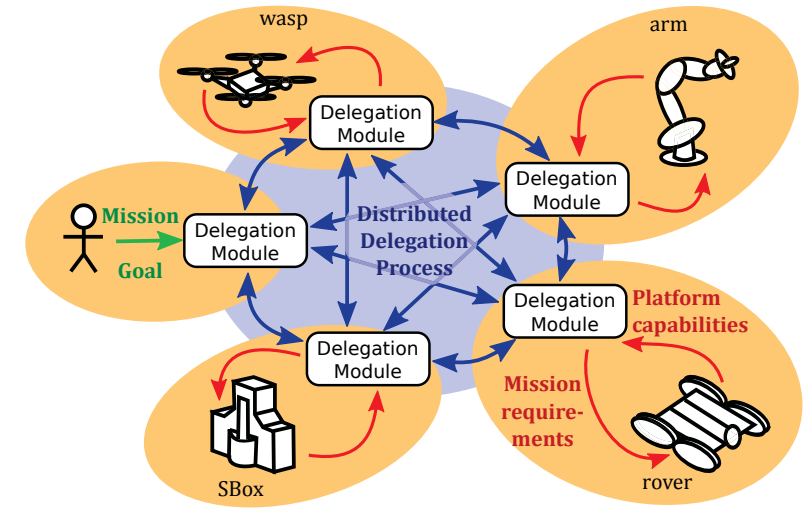

Fig. 2: Overview of the distributed delegation process and of the agents for the autonomous battery exchange during the SHERPA mission. Each agent has a delegation module that mediates between mission requirements and platform capabilities. The human operator can request or approve mission goals, but the system is also able to request, plan and execute missions completely autonomously without human supervision in a distributed delegation process, in which tasks are assigned to the most suited agents.

for the SHERPA mission, as described in [16], are designed to enable a team of robotic agents to cooperate in planning and executing complex and hierarchical tasks in a dynamic mission environment. An overview of the delegation process and the agents and components involved in the battery exchange are shown in Figure 2.

A generic task or mission goal, such as the exchange of the UAV's battery, is expressed in the form of a goal request Task Specific Tree (TST), i.e. a hierarchically structured description of the task. The internal nodes of a TST represent control statements for the task's execution, while leaf nodes represent domain specific functionalities. The transformation of a high-level request into a goal request TST takes place during the mission by dynamically instantiating generic TST templates.

The involved agents interact through their delegation modules, which contain a TST factory that can create TST nodes and link them to ancestors and descendants across agents, as well as a TST Executor Factory that provides platform specific functionalities for the execution of a task. The delegation module and interaction protocols are based on ROS, the Robot Operating System.

In the first phase of the delegation, a goal request TST is negotiated. When an agent requests a goal, the goal request TST is sent to its delegation module, where the distributed delegation process allocates each node of the tree to a suitable agent by means of an auction. The most suited bidder for a task is determined by solving the constraint problem belonging to a cost function, taking into account the mission requirements and platform capabilities.

The result of a successful delegation is an expanded collaborative plan TST, where all nodes have been allocated to the participating agents. This TST can then be executed in the second phase of the delegation. The autonomous execution of the battery exchange operation is described in Section IV-A as an example of such an expanded collaborative plan TST. 


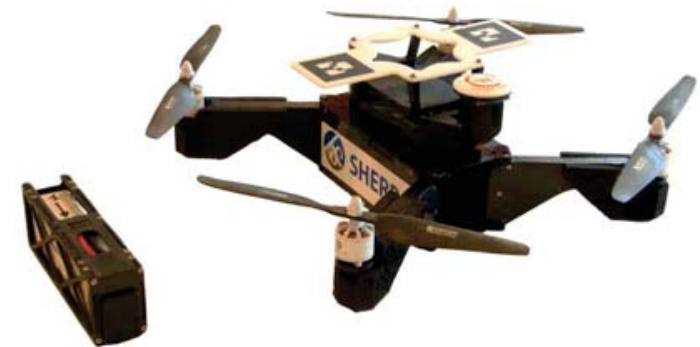

Fig. 3: The small scale UAV - the SHERPA wasp - and one of the exchangeable batteries

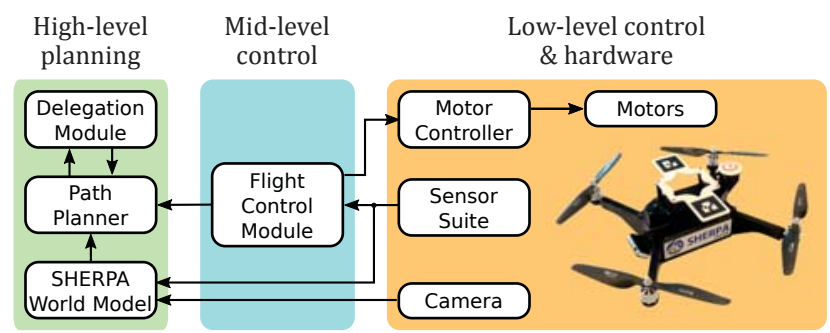

Fig. 4: System architecture of the SHERPA wasps.

\section{THE SHERPA SYSTEM}

In this section, the hardware and the control of the agents, i.e., the robots involved in the battery exchange, are described in detail. More specifically, the UAV (wasp), the SHERPA box (SBox) service station, the mobile ground rover, and the robotic arm are involved in the operation. In some cases the rover and arm can seen as a single agent for the higher-level planning, for instance when performing coordinated actions. They are then referred to as ground-rover-arm (GRA).

\section{A. Small-Scale UAVs - The SHERPA Wasps}

The role of the wasps, shown in Figure 3, is to gather data of the environment through cameras or other specialized sensors. Their high maneuverability and ability to hover over or follow targets makes them ideally suited for this task, even in otherwise inaccessible terrain. Due to their small size, low payload, and a flight time of approximately 20 minutes they are dependent on support from a ground station.

This reliance on a service station, and the requirement of a robust and efficient battery exchange mechanism, has resulted in a highly integrated interdependent design of the wasps and the SBox (ASLATECH, Italy). Rather than using a modular external battery carriage receptacle [9], [12], the battery bay is integrated into the structural frame of the wasps. This design leads to a stronger and lighter construction, while at the same time shielding the batteries and connectors from the environment. The battery carriage is made of a carbon fiber container that can slide in and out of the battery bays in the wasps and SBox. The UAV's main body furthermore contains the flight control module, a computer for high-level control, and a signal interface board, while the motor controllers are contained in the four arms holding the motors.

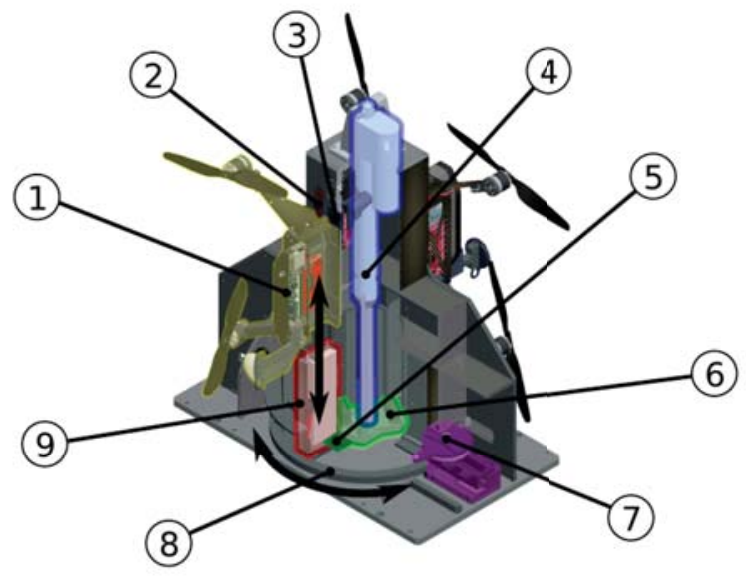

Fig. 5: A partial section view of the SBox and docked wasps showing the battery exchange mechanism. The wasp (1) is locked to the SBox with the clamps (2) when the switch (3) is engaged. To remove the battery (9) from the wasp a 'tongue' (5) engages the battery, which is pulled downwards into the battery magazine (8) when the linear actuator (4) moves the toolhead (6). To exchange the battery, the magazine is rotated by the actuator (7) which drives a Geneva mechanism. The battery exchange mechanism then places a charged battery into the wasp.

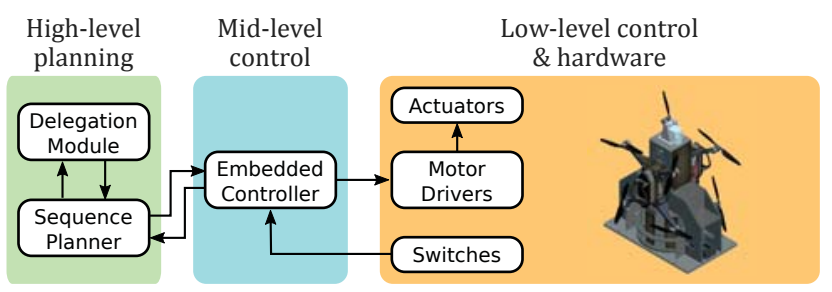

Fig. 6: System architecture of the UAV service station and communications hub - the SHERPA box.

As shown in Figure 4, the control of the wasps is organized in two main parts; a high-level planning module, and a midlevel flight control module. The high-level planner interacts with the delegation framework and includes a navigation path planner, which translates requested tasks into waypoints of suitable trajectories. The mid-level flight control is running on a real time operating system and interfaces the sensors and actuators. It runs stabilization algorithms that reject external disturbances such as wind, tracks the waypoints supplied by the path planner, arms and disarms the motors, and is capable of autonomous take-off and landing.

\section{B. Service Station - The SHERPA Box}

The SHERPA box is designed to function as the service station for docking and replenishing the wasps, as well as the computational and communications hub for the mission. The battery exchange mechanism itself consists of a linear actuator that slides the battery carriages from the UAV into a revolving battery magazine at the base of the SBox and vice versa. A sectional view of the SBox and the wasp is shown in Figure 5, displaying the components of the mechanism. Since the wasps do not have to land on the SBox, the battery exchange mechanism can be arranged vertically, which allows to service two wasps simultaneously.

For docking the wasp on the SBox, the wasp is initially 


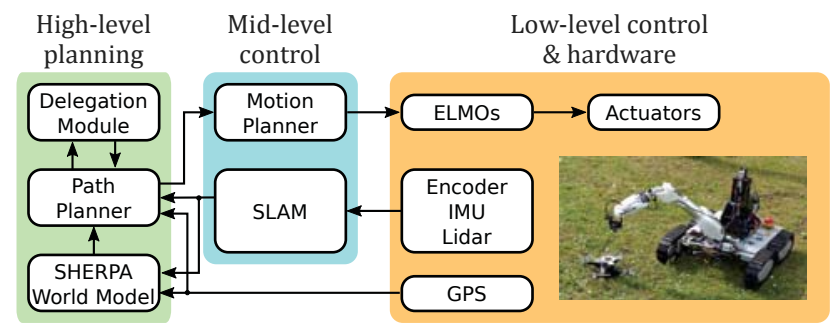

Fig. 7: System architecture of the mobile base station (ground rover).

placed on two small guide rails, before it is moved into its final docking position, guided by the rails and a short funnel. Once the wasp has been placed correctly, a switch registers that the wasp is docked, and two small clamps lock it securely in place. A tongue then extends from the toolhead at the end of the linear actuator, and engages a notch in the battery carriage, in order to move it downwards into a free slot of the battery magazine. The battery slots of the magazine are aligned with the battery compartment of the wasp by means of a Geneva mechanism, which rotates the magazine in discrete steps ensuring proper alignment, after the tongue has disengaged the empty battery. When the charged battery has been aligned, it is engaged by the tongue, and placed into the battery bay of the wasp, where it securely engages the electrical connectors and locks into place. The replenished wasp is then ready to be released by the SBox and to continue its mission.

As shown in Figure 6, the SBox has a hierarchal control structure. A high-level sequence planner and delegation module, which run on an Intel NUC, coordinate with the delegation framework. They translate requests for the Arduinobased embedded controller that interfaces with the sensors and actuators of the SBox.

\section{Mobile Base - The Ground Rover}

A specially developed (BlueBotics, Switzerland) ground rover serves as mobile base for the SBox and the robotic arm. It is characterized by its high degree of autonomy, endurance, and payload capacity. The rover is driven by four actuated tracks, that are mounted on a passively configurable chassis. These tracks allows the rover to traverse rough, mountainous terrain at a top speed of $0.8 \mathrm{~m} / \mathrm{s}$. Its range of sensors include a tilting laser scanner (lidar), IMU and GPS systems, as well as encoders for the tracks. The system is designed to run autonomously for three to six hours, depending on the usage of actuators. The battery can be changed without shutting down the hardware. The rover is furthermore equipped with an Intel NUC computer, Wi-Fi interface and internal power electronics.

Figure 7 shows the system architecture of the rover, which is divided into high-level planning, mid-level control, and low-level control and hardware. The high-level control interacts with the delegation framework, and formulates requested tasks as navigation problems that are solved by the path planner. A mid-level motion planner resolves the planned path and coordinates the individual motor drivers.

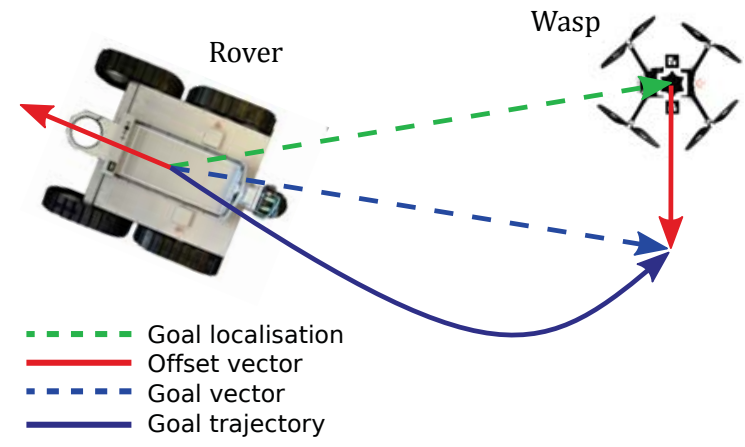

Fig. 8: Trajectory planning for the rover's approach phase through goal vector summation and planning algorithm. The goal localization is performed when the rover detects the wasp and adds it to the map. Adding the offset vector to that position results in the desired goal vector of the rover with the correct distance and orientation to the wasp. Finally a collision free trajectory is generated.

On the same level, a SLAM module combines data from the lidar, encoders, and IMU and provides a map of the environment with the rover's position to the high level path planner. The actual execution of the trajectory, and control of the actuators, is performed by ELMO Whistle digital servo drives (Elmo Motion Control Ltd., Israel).

During the battery exchange operation, the rover needs to plan and execute a trajectory that places the wasp within the workspace of the arm. It does so by using the map generated by the SLAM module, and the position of the wasp, provided by the arm and its end-effector camera. The GPS is not used for the precise localization of the wasp due to its limited accuracy. Using the relative body-inertial frame information provided by the SLAM algorithm, the wasp is localized in the map and its corresponding position is added to the map as an obstacle to ensure the safety of the systems. Figure 8 illustrates the summation of the position vectors that gives the rover's desired goal position as a function of the initial positions and orientations of the rover and wasp. The collision free trajectory is generated with an $\mathrm{A}^{*}$ algorithm implemented using a 2D navigation stack in ROS, and is subsequently executed by the platform.

\section{UAV Retrieval - The Robotic Arm}

The ground rover is equipped with a robotic arm [18], in order to robustly deploy and recover the wasps, and thus to facilitate the autonomous servicing and battery exchange operation. Figure 9 shows the system architecture of the arm.

The arm has 7 DOF and a reach of one meter, and is designed for a payload of $2 \mathrm{~kg}$. To enable compliant and safe interaction with the wasps and SBox during grasping and docking, the arm is equipped with two variable stiffness mechanisms in the shoulder and wrist joints. The arm's endeffector is a custom made gripper, that interlocks with an interface mounted on the wasp. As the arm is able to retrieve wasps without human assistance or requiring the wasps to perform precise and sensitive landing operations, it greatly extends the system's robustness and autonomy.

The high-level computational tasks are executed on an Intel NUC computer, while the low-level motor control is 


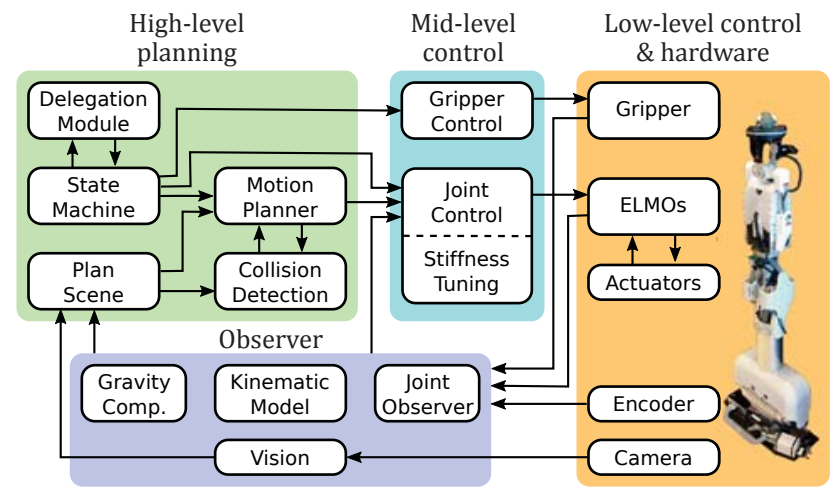

Fig. 9: System architecture of the arm.

performed by ELMO Whistle digital servo drives.

The arm's high-level planner includes a delegation module that interacts with the other agents, and a hierarchical finitestate machine (HFSM) based on the ROS decision making package, where the states of the HFSM are triggered by executors and represent the leaf nodes of the TST, i.e. the specific implementation of tasks for the arm. When a task requires the arm to move to a certain Cartesian goal, a suitable joint space trajectory is generated using the rapidly-exploring random tree (RRT*) algorithm implemented in MoveIt!. The mid-level joint controller then translates these trajectories to motor set-points and acts as supervisory controller.

The arm's observer combines data from the different sensors, including a Logitec c920 webcam mounted on the gripper, to obtain the state estimates. The vision-based localization of the wasps is also carried out by the arm observer. Markers placed on the wasp interface are detected using the ROS AR TRACK ALVAR wrapper. After its detection, a virtual wasp is placed in the plan scene of the arm, and its pose is published to other agents, such as the rover.

\section{EXPERIMENTAL RESUlts}

This section presents the autonomous battery exchange procedure and experimental results. The experiments were performed outdoors. The individual components of the tasks were carried out by the system's agents and coordinated by the delegation framework.

\section{A. Battery Exchange Operation}

The execution of a collaborative plan TST, i.e. the executor, is the second phase of the delegation process presented in Section II. The battery exchange operation is a relatively simple expanded collaborative plan TST, which could also be generated without the auctioning process, if the role of every agent is clear from the start. Here it thus serves as an example illustrating the structure of such a TST, while the full potential of the planning and delegation framework used here comes into play in more complex multi-agent operations. When the battery replacement executor, shown in Figure 10, is requested, it is delegated to the GRA agent and expands into several nodes that are delegated to the arm, wasp, rover, and SBox agents.

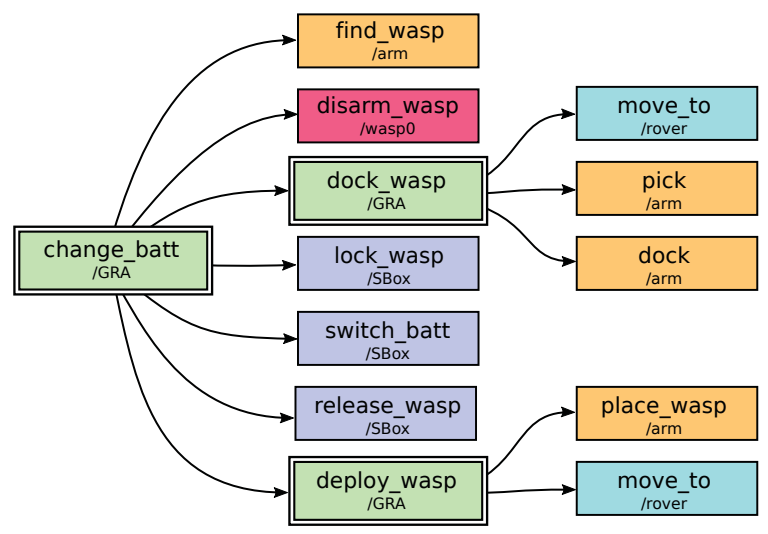

Fig. 10: The battery change executor (expanded collaborative plan TST) is delegated to the GRA agent. It expands into other executors, which are delegated to the arm, wasp, rover, and SBox agents. Internal nodes of the executor represent control statements, leaf nodes represent domain specific tasks. The different colors denote which agent the task is delegated to.

The change_batt executor is typically triggered by either the human operator, or fully autonomously by the UAV, which will request the battery exchange after landing when its power is running low. After accepting the delegation, the GRA expands the executor and commands the arm to localize the UAV through the find_wasp executor. This executor triggers the arm to search for the UAV with its end-effector camera through coordinated motions of the arm and the rover, after which the location and pose of the UAV is published to the other agents. Next, the UAV is commanded to disarm its motors, and the GRA to dock the UAV. The dock_wasp executor then in turn expands into the move_to, pick, and dock executors, which command the rover to approach the UAV, and the arm to grasp and move the UAV into the docking positing on the SHERPA box. The following lock_wasp, switch_batt, and release_wasp executors are delegated to the SBox, and lock the UAV in the docking position, replace the depleted battery for a charged one, and release the UAV respectively. Finally the UAV is deployed again by placing it on the ground, and moving the GRA away from it, such that the UAV and GRA can continue their respective missions.

\section{B. Experimental Results}

When the GRA is delegated to exchange the landed UAV's batteries, the arm determines its precise position relative to the rover. Figure 11 shows the detection of the UAV's markers as described in Section III-D. The still image of the camera feed is overlaid with the detected location of the marker, and the virtual collision object of the plan scene.

The virtual UAV is included the plan scene of the arm, as shown in Figure 12, and published.

The rover then plans a collision-free trajectory, as outlined in Section III-C, and approaches the UAV, such that the UAV is located within the arm's workspace and can easily be grasped. Figure 13 shows the rover and UAV locations in the map generated by the rover, and the rover's trajectory.

Figure 14 shows the arm docking and deploying the UAV as a sequence of still images. After approaching the UAV, 


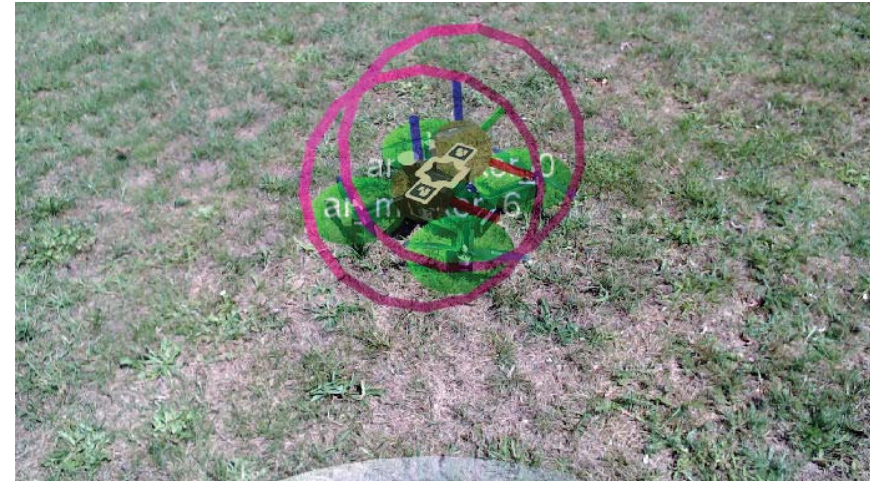

Fig. 11: The UAV is detected from the video feed of the arm's camera by means of the markers placed on the interface. The position of the markers is denoted by the two circles, while the orientation is shown through the superimposed coordinate frames. The virtual model of the UAV (shown in green) is also overlaid onto the live video stream.

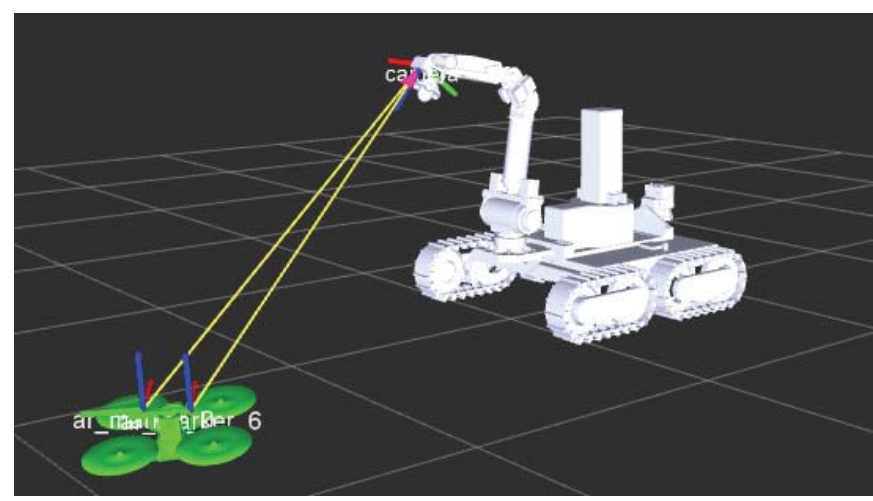

Fig. 12: The virtual collision object of the UAV is placed in the plan scene of the arm once it is detected. The UAV's position and orientation are also published to the other agents.

the arm picks up, grasps, and docks it on the SHERPA box, where the UAV's battery is exchanged according to Section III-B. The arm then places the UAV back on the ground and the rover moves away from the UAV so that it can continue its mission with a replenished battery. The tunable passive compliance of the arm has proven to be instrumental in the successful execution of the intricate interaction tasks. During grasping and docking of the UAV, the arm's compliance in the shoulder and wrist joints is controlled to make the operation more robust and reliable.

Figure 15 gives an overview of the execution of the battery exchange by presenting key variables of the rover, arm, and SBox over the course of the operation.

\section{CONCLUSiOns}

This paper presented the autonomous replacement of the battery of a small-scale UAV, in the scope of a search and rescue mission. The replacement is carried out by a heterogeneous team of robotic agents that are capable of planning and executing complex missions and tasks. This is facilitated by a software framework that is capable of coordinating a large group of diverse agents.

The successful battery exchange operation demonstrates these capabilities, and at the same time highlights how the endurance of small-scale UAVs can effectively be extended.

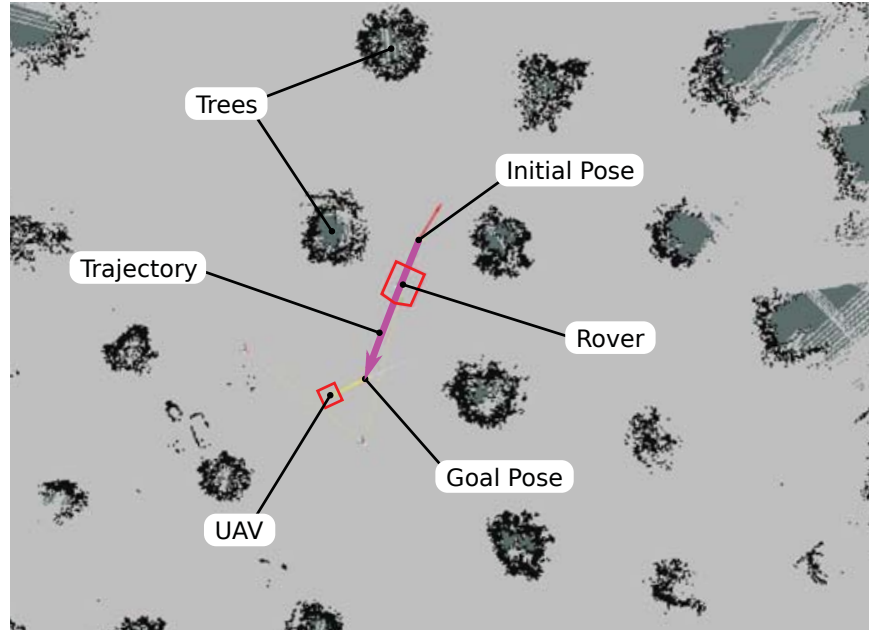

Fig. 13: The map generated by the rover during the experiments. Free cells are shown in light gray, occupied cells in black, and unknown cells in dark gray. The small trees in the rover's environment are clearly visible as obstacles. The map shows the initial pose of the rover and its goal pose, at an offset from the UAV position, as well as the generated trajectory connecting the two.

By replenishing its power supply, the UAV's flight time is greatly extend, as well as its operational radius, which is further increased by the service station mobility. The autonomy of the system is enhanced by the addition of a robotic arm to retrieve the UAVs, minimizing the need for human intervention during the mission. The main contribution of this work is the combination of a mobile service station and robotic arm to retrieve the UAVs.

\section{ACKNOWKEDGMEnTs}

The authors want to thank Andrea Sala and Dario Mengoli (ASLATECH, Italy, http://www.aslatech.com) for the mechatronic development of the SHERPA box and SHERPA UAVs, Pierre Lamon (BlueBotics SA, Switzerland, http://www.bluebotics.com) for the mechatronic development of the SHERPA rover, and Tommy Persson (Linköping University, Sweden) for the support in the implementation of the delegation framework.

\section{REFERENCES}

[1] L. Merino, F. Caballero, J. R. Martínez-de Dios, I. Maza, and A. Ollero, "An unmanned aircraft system for automatic forest fire monitoring and measurement," Journal of Intelligent \& Robotic Systems, vol. 65, no. 1, pp. 533-548, Jan 2012. [Online]. Available: https://doi.org/10.1007/s10846-011-9560-x

[2] L. Marconi, F. Basile, G. Caprari, R. Carloni, P. Chiacchio, C. Hurzeler, V. Lippiello, R. Naldi, J. Nikolic, B. Siciliano, S. Stramigioli, and E. Zwicker, "Aerial service robotics: The airobots perspective," in 2012 2nd International Conference on Applied Robotics for the Power Industry (CARPI), Sept 2012, pp. 64-69.

[3] L. Marconi et al., "The SHERPA project: Smart collaboration between humans and ground-aerial robots for improving rescuing activities in alpine environments," in Proceedings of the IEEE International Symposium on Safety, Security, and Rescue Robotics, 2012, pp. 1-4.

[4] G.J.M. Kruijff et al., "Designing, developing, and deploying systems to support human-robot teams in disaster response," Advanced Robotics, vol. 28, no. 23, pp. 1547-1570, 2014. [Online]. Available: http://dx.doi.org/10.1080/01691864.2014.985335 


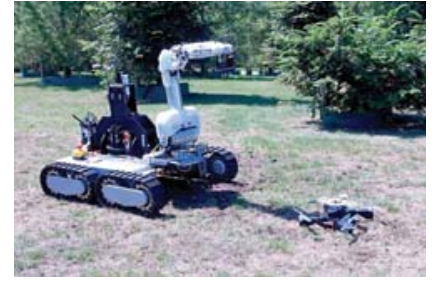

(a) Scan and approach

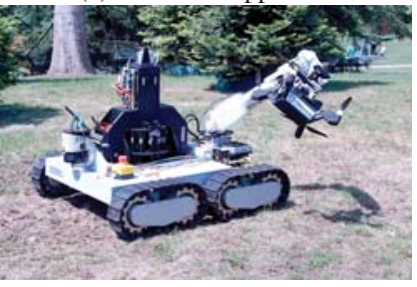

(c) Docking

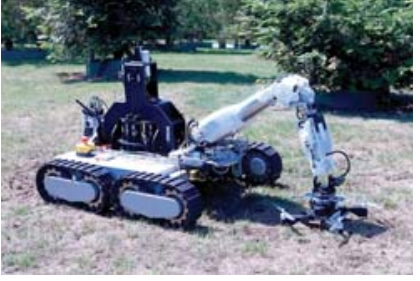

(e) Deploy

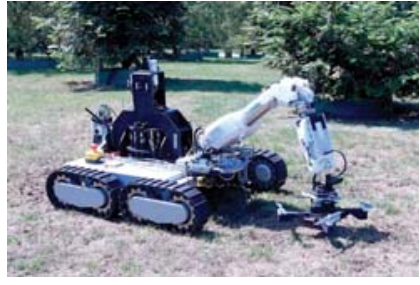

(b) Grasp

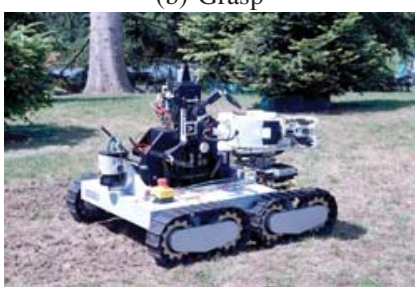

(d) Battery exchange

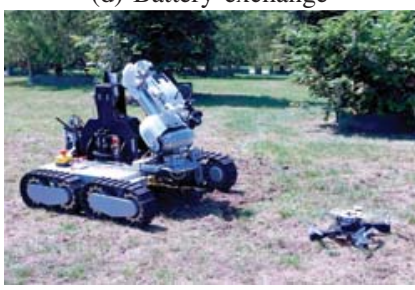

(f) Retreat
Fig. 14: After the ground rover approaches the landed wasp (Figure 14a) the arm picks it up (Figure 14b) and docks it on the SHERPA box (Figure 14c) where its battery is exchanged (Figure 14d). The arm then places it back on the ground (Figure 14e) and retreats away from the wasp (Figure $14 \mathrm{f})$.

[5] F. P. Kemper, K. A. O. Suzuki, and J. R. Morrison, "UAV consumable replenishment: design concepts for automated service stations," Journal of Intelligent \& Robotic Systems, vol. 61, no. 1, pp. 369-397, 2011.

[6] B. D. Song, J. Kim, J. Kim, H. Park, J. R. Morrison, and D. H. Shim, "Persistent UAV service: an improved scheduling formulation and prototypes of system components," in International Conference on Unmanned Aircraft Systems, 2013, pp. 915-925.

[7] D. R. Dale, "Automated ground maintenance and health management for autonomous unmanned aerial vehicles," Master's thesis, Department of Electrical Engineering and Computer Science, Massachusetts Institute of Technology, 2007.

[8] Y. Mulgaonkar, "Automated recharging for persistence missions with multiple micro aerial vehicles," Master's thesis, Department of Mechanical Engineering and Applied Mechanics, University of Pennsylvania, 2012.

[9] N. K. Ure, G. Chowdhary, T. Toksoz, J. P. How, M. A. Vavrina, and J. Vian, "An automated battery management system to enable persistent missions with multiple aerial vehicles," IEEE/ASME Transactions on Mechatronics, vol. 20, no. 1, pp. 275-286, 2015.

[10] K. A. Swieringa, C. B. Hanson, J. R. Richardson, J. D. White, Z. Hasan, E. Qian, and A. Girard, "Autonomous battery swapping system for small-scale helicopters," in IEEE International Conference on Robotics and Automation, 2010, pp. 3335-3340.

[11] K. A. O. Suzuki, P. Kemper Filho, and J. R. Morrison, "Automatic battery replacement system for uavs: Analysis and design," Journal of Intelligent \& Robotic Systems, vol. 65, no. 1, pp. 563-586, 2012.

[12] K. Fujii, K. Higuchi, and J. Rekimoto, "Endless flyer: A continuous flying drone with automatic battery replacement," in 2013 IEEE 10th International Conference on Ubiquitous Intelligence and Computing and 2013 IEEE 10th International Conference on Autonomic and Trusted Computing, Dec 2013, pp. 216-223.

[13] R. Godzdanker, M. J. Rutherford, and K. P. Valavanis, "Islands: a self-leveling landing platform for autonomous miniature UAVs," in IEEE/ASME International Conference on Advanced Intelligent Mechatronics, 2011, pp. 170-175.

[14] F. Bourgault, T. Furukawa, and H. F. Durrant-Whyte, "Decentralized

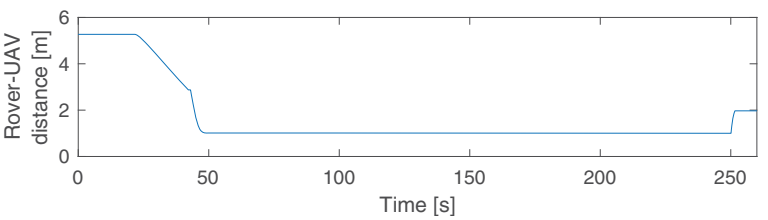

(a) Distance between rover and wasp.

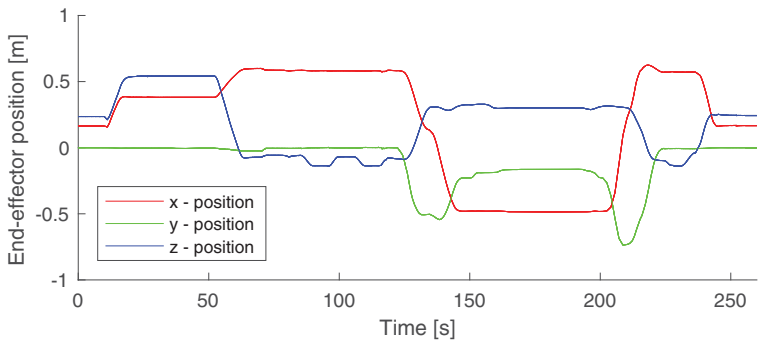

(b) End-effector position of the arm.
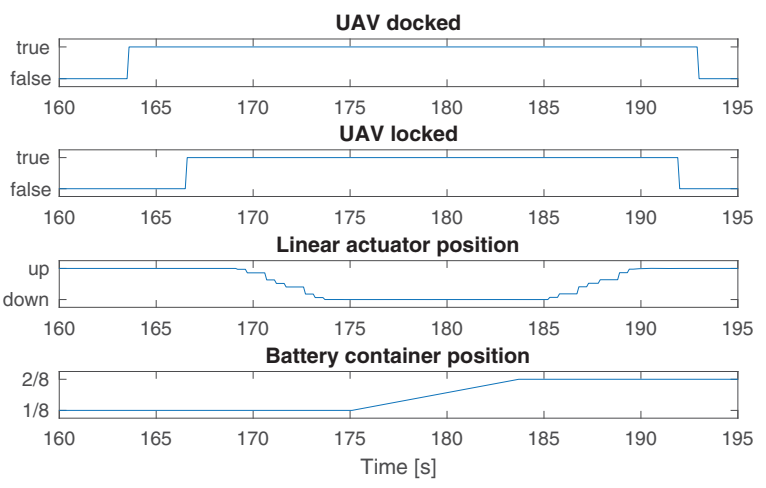

(c) Status of the SBox.

Fig. 15: Overview of the battery exchange operation through the status of the involved agents. Figure 15a shows the distance between the rover and wasp, as the rover approaches it during the execution of the dock_waspexecutor, and moves away from the wasp along the trajectory generated by the rover's path planner during the deploy_wasp-executor. Figure $15 \mathrm{~b}$ shows the end-effector position of the robotic arm. The arm moves first into a scanning pose during find_wasp and the start of dock_wasp, before it grasps and docks the wasp, and places it back on the ground during the deploy_wasp executor. Figure 15c shows the status of the SBox. The SBox registers the wasp as docked when the arm successfully places the wasp on the SBox. The wasp is then locked by the docking clamps while the linear actuator moves the empty battery into the battery container, which then moves a charged battery into position that is inserted into the wasp by the linear actuator.

bayesian negotiation for cooperative search," in IEEE/RSJ International Conference on Intelligent Robots and Systems, 2004, pp. 26812686.

[15] J. Gancet, G. Hattenberger, R. Alami, and S. Lacroix, "Task planning and control for a multi-UAV system: architecture and algorithms," in IEEE/RSJ International Conference on Intelligent Robots and Systems, 2005, pp. 1017-1022.

[16] P. Doherty, J. Kvarnström, P. Rudol, M. Wzorek, G. Conte, C. Berger, T. Hinzmann, and T. Stastny, "A collaborative framework for 3D mapping using unmanned aerial vehicles," in Proceedings of the 19th International Conference on Principles and Practice of Multi-Agent Systems (PRIMA), Phuket, Thailand, 2016, pp. 110-130.

[17] J. Kim, B. D. Song, and J. R. Morrison, "On the scheduling of systems of UAVs and fuel service stations for long-term mission fulfillment," Journal of Intelligent \& Robotic Systems, vol. 70, no. 1, pp. 347-359, 2013.

[18] E. Barrett, M. Reiling, G. Barbieri, M. Fumagalli, and R. Carloni, "Mechatronic design of a variable stiffness robotic arm," in Proceedings of the IEEE/RSJ International Conference on Intelligent Robots and Systems, 2017. 